\title{
Nutritional Support to Critically Ill Patient
}

\section{Iqbal Hossain Chowdhury*}

Associate Professor (Intensive Care Unit), Anaesthesia, Department of Analgesia, \& Intensive Care Medicine, BSMMU, Dhaka

* Corresponding author: iqbalhossain56@gmail.com

(Journal of BSA, 2010; 23(1): 30-33)

\section{Introduction}

Nutrition is defined as science of food and relationship to health. It is not a single science but a cluster of sciences related to the production and utilization of food ${ }^{1}$. Critical illness evoke a constellation of metabolic changes in the host including a transitory "ebb" phase followed by a hyper metabolic "flow" phase. Magnitude of the change is proportional to the extent of insult or illness. Those changes require an extra amount of energy in additional to basic metabolic requirement to maintain the nutritional status. If the basic and extra amount of energy cannot be provided, the patient may show diverse systemic functional impairments. Nutrients are needed for protein synthesis, for organ function and to sustain life. ${ }^{2}$ Critical illness is usually accompanied by anorexia or inability to eat because of impaired consciousness, sedation or intubation through upper airway. Patients are also metabolically stressed by the severity of the illness. Therefore, without nutritional support there is rapid loss of body weight and muscle mass. American Society for Perenteral and Enteral nutritional has included the following, ${ }^{2,3}$ (a) detection and correction of preexisting malnutrition, (b)prevention of progressive protein energy malnutrition, (c) optimizing patients metabolic state, and $(\mathrm{d})$ reduction of morbidity and time of convalescence. Nutritional support to critically ill patient in Intensive Care Unit (ICU) must follow the same rules as any other form of treatment with careful appraisal in each patient of likely benefit or harm to be expected from it. ${ }^{4}$

\section{Effects of Malnutrition}

The accelerated catabolism associated with acute illness or injury may further exacerbate tissue loss superimposed upon weight loss. Weight loss more than $8 \%$ results increasing impairment of function, handicap recovery from disease and multiply its complications. ${ }^{4}$ Malnourished persons suffer from muscle weakness and muscle fibers as well as respiratory muscles including diaphragm, impairing respiratory drive, ability to cough and clearing secretion. ${ }^{4,5}$ Malnutrition impaired immune function and increase rate of infection. 6,7,8 Acute illness and malnutrition also impair the digestive and barrier function of gut and may be protected by enteral feeding. Cardiovascular reflexes, vasoconstrictor responses to cold, heat conservation also affected by malnutrition. ${ }^{9}$ Malnutrition also contributes to increased surgical risk, poorer wound healing and slower recovery from surgery. ${ }^{4}$ Starvation and the responses to injury and immobility contribute to excess salt and water retention and negative nitrogen balance.

\section{Clinical Decision to Treat}

Nutritional support has been shown to be effective in improvement in nitrogen balance, wound healing, restore immune competence, facilitated weaning from ventilator and reduce mortality and morbidity in critically ill patients. ${ }^{3-4}$

From a consideration of the evidence outlined so far, the following indications for nutritional support are sugested. ${ }^{4}$

a. Weight loss greater than 10 percent and continuing.

b. Continuing inadequate oral intake.

c. The presence of the diseases whose known natural history is associated with accelerated catabolism and poor food intake for 10 days or more.

\section{Nutritional Assessment of Patient}

A normal nutritional status is a key element in the ability of a patient to overcome a critical illness. All the traditional markers of malnutrition lose their specificity in the sick adults as a number of non-nutritional factors may affect each. Nutritional 
assessment can be done by obtaining dietary history, clinical examination, anthropometry and laboratory investigations.

a. Dietary history: History includes dietary habits, nutrients intake, quality and quantity of food and omission of any major item may lead to malnutrition. Important questions include recent unintentional weight loss $(>10 \%)$, recent surgical stress, nausea, vomiting, diarrhoea, and the presence of comorbid illness.

b. Clinical examination: Signs of nutritional deficiency, such as weakness, muscle wasting, loss of subcutaneous fat, skin rashes, hair thinning, pallor, oedema, ascitis, fingernail abnormalities and many other clinical parameters come under practically feasible. Particular signs of specific nutrient deficiencies must be noticed.

c. Anthropometry: Anthropometric measurements are sufficient to define the nutritional status in healthy individuals but may be affected by non-nutritional factors. Therefore, anthropometric measurements must be interpreted with care. Measurement includes body weight, mid-arm circumference, mid-leg circumference, and triceps skin fold thickness etc.

d. Laboratory investigations: Investigations consists those indicate protein status and biochemical tests for micro nutrient deficiencies.

i. Haemoglobin estimation, ii. Serum total protein. iii. Serum albumin, iv. Lymphocyte count.

\section{Routes of Administration of Feed}

In deciding upon the rule of administration of feed, the rules are simple. If the gut works, try to use it. If the patient can swallow, try oral supplements or failing this, some form of enteral feeding by a fine-bore naso-gastric tube. Alternative enteral routes to oral feeding can be shown separately as under.
a. Naso-duodenal tubes,
b. Gastrostomy.
c. Jejunostomy tube or catheter-either feeding or percutaneous.

Enteral route of administration may be a good choice among the enteral and parenteral feeding in consideration of the complications of parenteral feeding and advantages of enteral feeding.

\section{Advantage of Enteral Feeding over Parenteral}

If the gastrointestinal tract is functional, the tube feeding is easier, safe and less costly than parenteral nutrition. It is possible that enteral feeds may also permit better utilization of nutrients, maintain mucosal integrity, and decrease the incidence of stress related haemorrhagic gastritis. ${ }^{11}$ It stimulates intestinal blood flow. Recent works also suggest that enteral nutrition may lead to reduction on mortality in patients ventilated for prolonged periods. ${ }^{3}$ Consequently, when spontaneous oral feeding is inadequate then feeding can be given to all patients except non-functioning gut cases. Enteral feeding has been shown to be gut protective and reduce the associated rise in hapatic enzyme in haemorrhagic shock or endotoxic shocked patient. ${ }^{2}$ Patients with blunt and penetrating trauma enteral feeding is better tolerated and associated with a lower frequency of infection within 24 hours. $^{2}$ Enteral delivery of nutrients compared to total per enteral nutrition (TPN) may reduce some complication in severely injured trauma patients and has been associated with a decrease in GIT mucosal permeability. ${ }^{12}$ Avoidance of immune suppression and the complications of central venous canula insertion required for parenteral feeding give additional advantage to entral feeding.

\section{Energy Expenditure and Calculation of Energy Requirement}

The key decision to provide nutritional support to critically ill patient involves the provision of adequate but not excessive amount of energy. Basal Metabolic Rate (BMR) can be estimated by heat loss using direct calorimetry, which is only possible in laboratory setting. In practice, caloric requirement is estimated by indirect calorimetry, which measures oxygen consumption, and energy expenditure is calculated. Formula like HarrisBenedict equation or other simpler more practical formula can be used to predict the basic energy expenditure. ${ }^{13}$ Another 500-1000 kcal should be added for the hyper catabolic state of critically ill patients. 


\section{Feed Composition}

There has been considerable effort to determine what constitute adequate and optional nutritional support to critically ill patient. Enteral nutritional supplement should be composed of optimal combination of protein, carbohydrate and fat. The volume, water content, ionic composition and addition of trace elements and vitamins are of great importance. They can be prepared from fresh foods or commercially prepared diets. Many prepared feeds in liquid or powder form are commercially available. These vary in their protein, carbohydrate and fat source, electrolyte, mineral, vitamin content, osmolality and contents of specific nutrients including fiber, branched chain-1 acid (BCAA), essential amino acid, glutamine, arginine, neucleotides and other nutrients. Lactose-free, isotonic liquid feed providing approximately two-third of non-protein energy as carbohydrate meets the need of most patients. When necessary, such a feed can be modified by the addition of individual carbohydrate, protein or fat sources to meet specific need.

\section{Complications Associated with Enteral Feeding}

a. Complications associated with feeding tube.

i. Trauma and bleeding.

ii. Gastric or bowel perforation.

iii. Tube obstruction.

iv. Tube displacement.

v. Patient discomfort.

b. Complications related to enteral feeding,

i. Nosocomial infection from bacterial contamination.

ii. Nausea, abdominal distension and discomfort.

iii. Regurgitation or vomiting

iv. Pulmonary aspiration of feed.

v. Diarrhoea.

vi. Intestinal pseudo-obstruction.

c. Complications related to feed content.

i. Hyper and hypoglycaemia.

ii. Glucose intolerance.

iii. Azotaemia. iv. Hypercarbia.

v. Electrolyte abnormalities.

vi. Specific deficiency disorder with long term use.

\section{Monitoring of Patients Receiving Enteral Feeding}

a. Clinical

i. Examine the abdomen for distention and bowel sounds.

ii. Record the frequency and consistency of stool, and their colour, odour and estimated weight or volume.

iii. Note patient's complaints of fullness, nausea, vomiting, abdominal pain or tenderness.

b. Blood

i. Measure blood glucose, blood urea nitrogen and serum electrolyte levels at least twice a week or more frequently if they are abnormal.

ii. Measure SGOT, SGPT, LDH, serum albumin, bilirubin, calcium, magnesium and phosphate levels once week.

iii. Measure serum triglyceride and cholesterol levels at least once a week in patients receiving fat in their diets.

c. Urine

Test for glucose for 6 hours and cover with crystalline insulin as follows: $5 \mathrm{U}$ for $0.23 \mathrm{~g} / \mathrm{dl}, 15 \mathrm{u}$ for $1 \mathrm{~g} / \mathrm{dl}$ and $20 \mathrm{U}$ for $2 \mathrm{~g} / \mathrm{dl}$.

Repeat the test hourly for $2 \mathrm{~g} / \mathrm{dl}$ and cover with $20 \mathrm{U}$ for $2 \mathrm{~g} / \mathrm{dl}$ every 2 hours. Inform physician if glycosuria lasts for 4 consecutive hours. Then resume feeding with a lesser rate or with a formula containing less carbohydrate.

Present Status of Nutritional Support to Critically ill Patient in Bangladesh

The method of nutritional support to critically ill patient in different hospitals of Bangladesh is in primitive state. Because we are running shortage of specialist dietetics, lack of knowledge, and other resources etc. There is no organized way of providing nutritional support; moreover, it is difficult to specify a food composition without adequate knowledge about nutrition. As a result present trend is that either the clinician prescribe 
a branded preparation or patients party prepare a homemade preparation at there own without proper calculation about the patients energy requirement. Some times doctors become delayed to start the nutritional support to patient even after several days of admission in hospital. So there are many scopes remain to work in this area for better improvement.

\section{Conclusion}

Patient undergoing treatment in ICU remains in critical state of their health, where provision of nutritional support can ensure a good organ function or cat not prevent their functional impairment. Method of nutritional support may vary from individual to individual considering nature of disease process. A team of personnel, which should include clinician, nutritionist, dietetics and cools, should do it. Problem may arise due to lack of coordination between the members of treating committee or absence of such committee. As nutritional support to critically ill patient is an obvious life support; it should be ensured with proper importance and dedication.

\section{References}

1. Tod Hunter EN. Historical landmark in Nutrition. 5th ed, Washington DC Nutrition foundation 1984 .

2. Andrew RW, Marc JS, Mervyn S, Peter MS. Oxford Text Book of Critical Care. 1st ed. Oxford: Oxford University Press, 1999: 394.

3. TE Oh. Intensive Care Manual. 4th ed. Oxford: Butterworth- Heinemann; 1998; 716-26.
4. Th E J Healy, Peter J Cohen. A practice of Anaesthesia. 6th ed. London: Wylie and hurchill-Davidson; 1995; 886-896.

5. Doekel Jr RC, Zwillich CW, Scroggin CH. Clinical semi-starvation: depression of hypoxic ventilatory response. New England Journal of Medicine 1976;295:358-361.

6. Shizgal HM. Nutrition and immune function. Surgery Annals 198 1; 12:15-29.

7. Chandra RK. Immunity and infection. In: Kinney JM, Jeejeebhoy KN, Hill GL, Owen OE, eds. Nutrition and metabolism in patient care. Philadelphia; WB Saunders, 1988; 598-604.

8. Windsor .IA, Hill GL. Risk factors for postoperative pneumonia: the importance of protein depletion. Annals of Surgery 1988; 208:209-14.

9. Macdonald IA, Bennett T, Sainsbury R. The effect of a 48 hour fast on the thermoregulatory responses to garded cooling in man. Clinical Science 1985; 67: 445-52.

10. Al Maliki T, Langer JC, Thompson V, et al. A prospective evaluation of the button gastrostomy in children. Can Surg 1991; 10: $18-22$.

11. Frisancho AR: New norms of upper limb fat and muscle areas for assessment of nutritional status. Am J Clin Nutr 1981; 34: 2540.

12. Jesse BH, Gregory AS, Lawrence DH. Wood, Principles of Critical Care. 2nd ed. New York: Mc Graw-Hill. 1998; 203.

13. Park K. Park's Text Book of Preventive and Social Medicine. 19th ed, India. M/S Banarsidas Bhanot Publishers 2007; 501-507. 